\title{
RESER VATION STATUS AND PRIORITIES FOR TASMANIAN PLANTS I. ANGIOSPERMAE (DICOTYLEDONAE)
}

\author{
by J.B. Kirkpatrick, L. Gilfedder, F. Duncan and Stephen Harris
}

(with two text-figures and three tables)

Almost one-fifth of Tasmanian native dicotyledonous angiosperms are not known from any national park or equivalent reserve. Extinct, endangered, vulnerable and unreserved species are most common among annuals and least common among woody plants. The unreserved species have their distributions concentrated between Launceston and Hobart in the dry, naturally grassy Midlands. A minimum reservation strategy is suggested for those species for which this option still exists.

Key Words: dicotyledonous angiosperms, reservation status, reservation strategy, Tasmania.

In BANKS, M.R.et al. (Eds), 1991 (31:iii): ASPECTS OF TASMANIAN BOTANY - A TRIBUTE TO WINIFRED CURTIS. Roy. Soc. Tasm. Hoban: 163-172.

\section{INTRODUCTION}

The last quarter-century has seen a massive loss of Tasmanian natural vegetation to pasture, plantation and impoundment (Kirkpatrick 1988). While Tasmania has $22 \%$ of its land surface within secure national parks and equivalent reserves, recent botanical surveys, particularly in lowland dry sclerophyll vegetation, indicate that a large proportion of Tasmanian native plant species are either totally unreserved or poorly reserved (Brown et al. 1977, Kirkpatrick et al. 1980, Brown et al. 1983, Duncan 1985, Kirkpatrick et al. 1988a,b). The most recent list of unreserved and poorly reserved higher plant species for Tasmania is that of Duncan (1985). A much-expanded information base, with new herbarium records and the records from recent major vegetation surveys, has made a new analysis appropriate, and there is a need for information on the appropriate locations for species reservation.

In this paper we assess the reservation status of all Tasmanian native dicotyledonous angiosperm species listed in Buchanan et al. (1989) and undertake a distributional analysis to indicate the most appropriate areas for reservation, where reservation is still possible. We also classify the unreserved and poorly-reserved species into the conservation status classes of Briggs \& Leigh (1988) from a purely Tasmanian perspective.

\section{METHODS}

In order to be considered adequately reserved under this classification, a species had to occur in at least one large population (if not a Tasmanian endemic) or two large and separated populations (if an endemic) within reserves that require the permission of both Tasmanian Houses of Parliament for revocation (i.e. national park (NP), state reserve (SR), nature reserve (NR), aboriginal site, historic site (HS), or game reserve) or within the Western Tasmania World Heritage Area (WHA), which is protected under an international convention. Forest reserves, state recreation areas, protected areas and conservation areas without statutory management plans do not yet enjoy the security of tenure of the above reserves. The level adopted for non-endemic species assumes that species will be reserved in part of their range outside Tasmania. The level of reservation adopted as sufficient in this study is less than minimal in the context of the importance of preservation of the genetic variability of species. It has been adopted to allow easy identification of major reservation deficiencies and major reservation requirements.

To determine which species fulfilled the reservation criteria, the following sources were used:

- quadrat data sets for grassy ecosystems (Kirkpatrick et al. 1988a), wet eucalypt forests (Kirkpatrick et al. 1988b), alpine vegetation (Kirkpatrick 1986), wetlands (Kirkpatrick \& Harwood 1983) and heath (Kirkpatrick 1977);

- conservation status assessments of Brown et al. (1983), Jarman et al. (1984, 1988), Duncan \& Brown (1985) and Duncan (1985);

- recently published and unpublished species lists for reserves or parts of reserves (e.g. Brown \& Bayly-Stark 1979a,b, Harris \& Kirkpatrick 1982);

- specimens in the Herbarium of the Tasmanian Museum and Art Gallery (HO) and the National Herbarium in Sydney and Melbourne.

Records earlier than 1970 were not accepted as sole evidence of reservation. In most cases the areas covered 
by such records have been revisited by one of the authors since 1970 .

Each of the unreserved taxa was mapped on the $10 \times 10 \mathrm{~km}$ National Mapping grid square system. These data were used to construct a map of unreserved species concentration (fig. 1) and to conduct an iterative analysis of potential reserve locations following the method of Kirkpatrick (1983), with endemic species requiring two reserves, other species one reserve, and endangered and vulnerable species scoring 3 and 2 respectively, while other species scored 1. In the first instance of reservation, endemic species scored 6, 4 and 1 respectively. Putatively extinct species were excluded from the analysis.

Status as extinct, unknown, endangered or vulnerable was determined following the general rules of Briggs \& Leigh (1988) within the restricted context of Tasmania. Chi-squared was used to test for concentrations of elements of species in each of the classes and for lack of reservation in general.

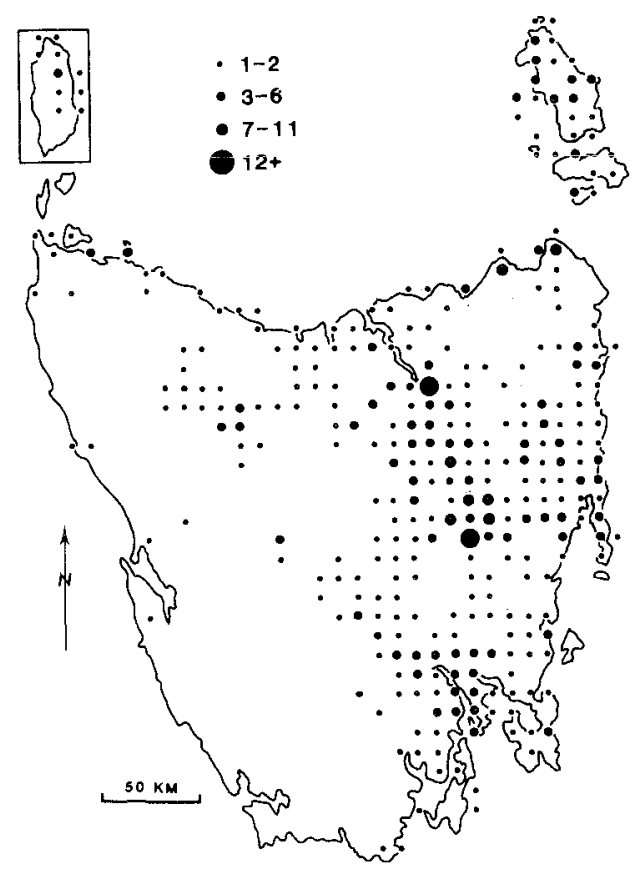

FIG. 1 - Numbers of unreserved dicotyledonous angiosperms in $10 \times 10 \mathrm{~km}$ National Mapping grid squares.

\section{RESULTS AND DISCUSSION}

Of the native angiosperms under discussion, 168 species are not known to occur within the state reserve system, while a further 19 are inadequately reserved endemic species (table 1, see pp.167-171). These constitute $19.9 \%$ of the total number of native dicotyledonous species in Tasmania (Buchanan et al. 1989) and include 36 in the Asteraceae, 17 in the Fabaceae, and seven to ten in each of the Myrtaceae, Epacridaceae, Rhamnaceae, Scrophulariaceae, Brassicaceae, and Haloragaceae (table 1). Of the families with more than seven native species, the Rhamnaceae and Haloragaceae have more than $30 \%$ of their native species in table 1 .

The species fall into several distributional classes. Many have the southern parts of their range in the Bass Strait islands or northern Tasmania. Many more are confined to the grassy ecosystems of Tasmania. There are concentrations of unreserved species on the central east coast of Tasmania and in the Midlands, although in absolute terms the Midlands has the most species (fig. 1).

There is a concentration of annual species within the extinct, endangered and vulnerable classes compared to the flora as a whole and the unreserved species as a whole (table 2 , see p. 173). Woody plants would seem to be least prone to endangerment and extinction in Tasmania, but this phenomenon may be due to the fact that small herbs and annuals are more often overlooked or not identified in vegetation surveys. The extinct species (which include species that have been described for Tasmania then "lost", and may still be rediscovered) are either microendemics, with an original range less than $100 \times 100 \mathrm{~km}$, or grassy ecosystem species that extend to the mainland, where most survive, albeit in a parlous state.

Figure 2 and table 3 (see p. 174) show areas indicated by the iterative analysis as being most appropriate for reservation. Many of these areas have been recommended for reservation in previous studies. Among the top eight, three are in the Midlands, and two are in northern Tasmania, while one is in the northwest and two are on the central east coast. Many of these localities are also known to be important for the conservation of monocotyledonous angiosperms and plant communities. The importance of the natural remnants in the Midlands is emphasised by the recommendations in table 3 . As previously noted (Fensham \& Kirkpatrick 1989) the opportunities for reservation are rapidly declining as clearance progresses. Adequate reservation for many species could be achieved by providing, through legislation, appropriate security of tenure for existing inadequately protected reserves (e.g. forest reserves, protected areas, most conservation areas). Other areas identified in this study 
have good prospects for secure reservation at the time of writing (December 1989). Completion of more thorough botanical surveys in state reserves is likely to reduce the list of species considered to be unreserved or poorly reserved in Tasmania, particularly if surveys are directed towards reserves in the east of the state. The priorities for survey include Coal River Gorge NR, St Patricks Head SR and the non-plateau portion of the Ben Lomond NP.

The data and recommendations presented in this paper cannot be considered the final word on the reservation of dicotyledonous plants in Tasmania. Our taxonomic and distributional knowledge constantly changes. However, we are confident that the pattern of proposed reservation will withstand the test of time.

\section{ACKNOWLEDGEMENTS}

We gratefully acknowledge the help of Rod Fensham, Fiona Coates, Mick Brown, Jill Hickie, Adrian Pyrke and the staff of the Herbarium of the Tasmanian Museum and Art Gallery (Hobart and Launceston), the National Herbarium of NSW and the National Herbarium of Victoria. Dr G. van der Geer drew the figures.

\section{REFERENCES}

BriggS, J.D. \& LeIGH, J.H., 1988: Rare or threatened Australian plants. Aust. Nat. Parks Wildl. Serv. Spec. Publ. 14.

Brown, M.J. \& BAYlY-STARK, H.J., 1979a: Vegetation of Maria Island. Tasm. Nat. Parks Wildl. Serv., Wildl. Div. Tech. Rep. 79/1.

Brown, M.J. \& Bayly-Stark, H.J., 1979b: The plant communities of the East Risdon Nature Reserve. Tasm. Nat. 58: 1-11.

Brown, M.J., Hoggins, D.D. \& Bayly-STark, H.J., 1977: Conservation of flora in Tasmania. Tasm. Nat. Parks Wildl. Serv., Tech. Rep. 77/4.

Brown, M.J., KirkPatrick, J.B. \& Moscal, A., 1983: AN ATLAS OF TASMANIA'S ENDEMIC FLORA. Tasmanian Conservation Trust, Hobart.

Buchanan, A.M., McGeary-Brown, A. \& Orchard, A.E., 1989: A census of the vascular plants of Tasmania. Tasm. Herb. Occ. Publ. 2.

DunCAN, F., 1985: Tasmania's vegetation and its response to forest operations. Working Paper 6 ENVIRONMENTAL IMPACT STATEMENT INTO THE TASMANIAN WOODCHIP EXPORTS BEYOND 1986. Tasmanian Forestry Commission, Hobart.
Duncan, F.D. \& Brown, M.J., 1985: Dry selerophyll vegetation in Tasmania. Tasm. Nat. Parks Wildl. Serv., Wildl. Div. Tech. Rep. 85/1.

Fensham, R.J. \& KIRKPATRICK, J.B., 1989: The conservation of original vegetation remnants in the Midlands, Tasmania. Pap. Proc. R. Soc. Tasm. 123: 229-246.

FiRTH M.J., 1969: The flora of Rocky Cape. Rec. Q. Vic. Mus. 3: $1-16$.

Harris, S. \& KirKPatrick, J.B., 1982: The vegetation of Schouten Island, Tasmania. Pap. Proc. R. Soc. Tasm. 116: 117-141.

Jarman, S.J., Brown, M.J. \& Kantvilas, G., 1984: RAINFOREST IN TASMANIA. Tasmanian National Parks and Wildlife Service, Hobart.

Jarman, S.J., Kantvilas, G. \& Brown, M.J., 1988: Buttongrass moorland in Tasmania. Tasmanian Forest Res. Counc. Inc. Res. Rep. 2.

KiRKPATRICK, J.B., 1977: THE DISAPPEARING HEATH. Tasmanian Conservation Trust, Hobart.

KIRKPATRICK, J.B., 1983: An iterative method for establishing priorities for the selection of Nature Reserves: an example from Tasmania. Biol. Conserv. 25: 127--134.

Kirkpatrick, J.B., 1986: Conservation of plant species, alliances and associations of the treeless high country of Tasmania. Biol. Conserv. 37: 43-57.

Kirkpatrick, J.B., 1988: Heritage and development in Tasmania. Aust. Geog. 19: 46-63.

Kirkpatrick, J.B., Brown, M.J. \& Moscal, A., 1980: THREATENED PLANTS OF TASMANIA'S EAST COAST. Tasmanian Conservation Trust, Hobart.

Kirkpatrick, J.B., Gllfedder, L. \& Fenshà, R., 1988a: CITY PARKS AND CEMETERIES: TASMANIA'S REMNANT GRASSLANDS AND GRASSY WOODLANDS. Tasmanian Conservation Trust, Hobart.

KiRKPATRICK, J.B. \& HaRwood, C.E., 1983: The conservation of Tasmanian wetland macrophytic species and communities. Pap. Proc. R. Soc. Tasm. 117: 5-20.

Kirkpatrick, J.B., Peacock, R.J., Cullen, P.J. \& Neyland, M.G., 1988b: THE WET EUCALYPT FORESTS OF TASMANIA. Tasmanian Conservation Trust, Hobart.

(accepted 10 August 1990)

J.B. Kirkpatrick and L. Gilfedder

Department of Geography and Environmental Studies, University of Tasmania, GPO Box 252C, Hobart, Tasmania, Australia 7001

F. Duncan

Forestry Commission, GPO Box. 207B, Hobart, Tasmania, Australia 7001

S. Harris

Department of Parks, Wildlife and Heritage, GPO Box 44A, Hobart, Tasmania, Australia 7001 
TABLE 1

Poorly Reserved Endemic and Unreserved Dicotyledonous Plant Species*

\begin{tabular}{|c|c|c|c|c|}
\hline Species & $\begin{array}{c}\text { Conservation } \\
\text { status } \dagger\end{array}$ & $\begin{array}{c}\text { Distri- } \\
\text { bntion } \neq\end{array}$ & Lifeform $\$$ & $\begin{array}{c}\text { Proposed } \\
\text { reserve } \\
\text { locationף }\end{array}$ \\
\hline
\end{tabular}

Amaranthaceae

Alternanthera denticulata

Ptilotus spathulatus

Apiaceae

Eryngium ovinum

Hydrocotyle capillaris

H. comocarpa

H. laxiflora

Asteraceae

Bedfordia arborescens

Brachyglottis brunonis

Brachyscome parvula

B. perpusilla

$B$. sieberi var gunnii

B. radicata

B. rigidula

Calocephalus citreus

C. lacteus

Cotula vulgaris

Helichrysum lycopodioides

H. obtusifolium

H. pleurandroides

$H$. selaginoides

H. spiceri

Helipterum albicans

$H$. anthemoides

$H$. australe

$H$. demissum

Isoetopsis graminifolia

Leptorhynchos elongatus

Millotia tenuifolia

Odixia achlaena

Podotheca angustifolia

Rutidosis multiflora

Senecio macrocarpus

S. orarius

S. squarrosus

S. vagus

S. velleoides

Taraxacum cygnorum

Vittadinia australasica

$V$. cuneata

V. gracilis

V. megacephala

V. muelleri

Bignoniaceae

Pandorea pandorana

Boraginaceae

Myosotis exarrhena

Brassicaceae

Ballantinia antipoda

Barbarea australis
$\mathrm{X}$

E

V

H

H

H

$\mathrm{AH}$

AH

$\mathrm{H}$

T

le

P

$\mathrm{X}$

$\mathrm{X}$

e

K

$\mathrm{P}$

$\mathrm{X}$

$\mathrm{X}$

E

V

E

E

E

E

P le

X

$\mathrm{X}$

K

V

V

K

$\mathrm{X}$

E

$\mathrm{X}$

E

K

$\mathrm{X}$

$\mathrm{X}$

le
$1,2,12,23$

12

4

42

23

11

18,68

4,55

4

$10,15,49$

1,12

$-$

1,9

4,46

6,7

-

31

$-$

$-$

3

3

2

70

1

35

4,12

51

$-$

2,4

$-$

$\mathrm{AH} / \mathrm{H}$

$\mathrm{H} \quad 14$

$\mathrm{H} \quad 21,47$

$\mathrm{H}-$

$\mathrm{H} \quad-$

$\mathrm{H} \quad 1,23$

$\mathrm{H} \quad 1$

$\mathrm{H} \quad-$

$\mathrm{H} \quad 1,12,23$
CS 43

$\mathrm{H}$

AH

$\mathrm{AH} / \mathrm{H}$ 
Species

\section{Conservation \\ status $\dagger$}

Distri-

butionł
Lifeform $\S$

Proposed

reserve

location 1
Hutchinsia tasmanica

Lepidium desvauxii

L. hyssopifolium

L. pseudotasmanicum

Stenopetalum lineare

Brunoniaceae

Brunonia australis

Callitrichaceae

Callitriche umbonata

Campanulaceae

Lobelia pratioides

Pratia irrigua

Caryophyllaceae

Scleranthus diander

S. fasciculatus

Stellaria caespitosa

Chenopodiacae

Atriplex suberecta

Chenopodium erosum

C. pumilio

Convolvul aceae

Calystegia marginata

C. sepium

C. soldanella

Wilsonia humilis

W. rotundifolia

Crassulaceae

Crassula exserta

C. pedicellosa

Cucurbitiaceae

Sicyos australis

Dilleniaceae

Hibbertia obtusifolia

$H$. calycina

H. rufa

Epacridaceae

Acrotriche cordata

Epacris acuminata

E. apsleyensis

E. curtisiae

E. exserta

E. virgata

Leucopogon esquamatus

L. lanceolatus

Pentachondra ericaefolia

Euphorbiacae

Bertya rosmarinifolia

Fabaceae

Acacia axillaris

A. pataczekii

A. retinodes

Bossiaea ensata

B. obcordata

Desmodium varians var. gunnii

Glycine latrobeana

Gompholobium ecostatum

Goodia pubescens le

V

E

V

V

V

$\mathrm{V}$

V

V

K

K

$\mathrm{V}$

K

V

K

K

K

K

V

K

E

$\mathrm{X}$

le

$\mathrm{AH}$

$\mathrm{AH}$

$\mathrm{H}$

AH

$\mathrm{AH}$

H

$\mathrm{AH}$

$\mathrm{H}$

$\mathrm{H}$

27,28

H 1

$\mathrm{H}$

AH

$\mathrm{H}$

AH

AH

17,58

19

17,23

26

16

48

H 11

H $\quad-$

$\mathrm{H} \quad 50$

S 4

$\mathrm{S}$

AH

$\mathrm{AH}$

AH

$\mathrm{S}$

S

$\begin{array}{cc}\text { P } & \text { e } \\ \text { P } & \text { le } \\ & \text { le } \\ \text { P } & \text { le } \\ \text { PV } & \text { e }\end{array}$

$\mathrm{c}$

V

V

c

e

K

E

V

V
46

69

$1,4,11$

$-$

41

18

10

52

25,39

5

11,43

53

36,67

54

$6,22,24$

13,49

55

13

15

$2,4,16,54$

14

31 
Species

\section{Conservation status $\dagger$}

\section{Distri- bution \\ Lifeform}

\section{Proposed \\ reserve \\ location}

Hardenbergia violacea

Hovea lanceolata

Pultenaea hibbertioides

$P$. humilis

$P$. palacea

P. prostrata

$P$. selaginoides

Viminaria juncea

Geraniaceae

Pelargonium littorale

Goodeniaceae

Goodenia amplexans

G. barbata

Scaevola aemula

$S$. albida

S. calendulacea

Velleia paradoxa

Haloragaceae

Haloragis aspera

$H$. heterophylla

H. myriocarpa

Myriophyllum crispatum

M. glomeratum

$M$. integrifolium

M. muelleri

Lamiacae

Lycopus australis

Mentha australis

Prostanthera cuneata

$P$. rotundifolia

Westringia angustifolia

Lauraceae

Cassytha pedicellosa

Lentibulariaceae

Utricularia australis

Loganiaceae

Mitrasacme paradoxa

Lythraceae

Lythrum salicaria

Malvaceae

Gynatrix pulchella

Menyanthaceae

Nymphoides crenata

Villarsia exaltata

Monimiaceae

Hedycarea angustifolia

Myoporaceae

Myoporum parvifolium

Myrtaceae

Callistemon paludosus

Eucalyptus barberi

E. perriniana

E. morrisbyi

E. risdonii

E. rubida

Melaleuca pustulata
V

K

V

E

$\mathrm{V}$

E

PV

E

K

$\mathrm{X}$

$\mathrm{X}$

$\mathrm{V}$

V

K

$\mathrm{V}$

V

V

K

K

$\mathrm{V}$

$\mathrm{X}$

K

$\mathrm{X}$

V

P

$\mathrm{X}$

V

V

$\sqrt{2}$

$\mathrm{X}$

le

56

57

2, 16

4

1

10

38

$\mathrm{H}$

S

$\mathrm{S}$

$\mathrm{H}$

$\mathrm{H}$

$\mathrm{H}$

$\mathrm{H}$

30

$-$

$\mathrm{H} \quad 16$

$\mathrm{H} 2$

$\mathrm{H} \quad 45$

$\mathrm{H}$

H

H

$\mathrm{H}$

4,11

S

$\mathrm{H}$

S

$-$

25

21

$\mathrm{H}$

$\mathrm{AqH}$

39

AH

59

$\mathrm{AH}$

63

S

4, 11

$\mathrm{AqH}$

$2,4,16$

$\mathrm{AqH}$

63

T

60

S

55

P

10,24

$\mathrm{S}$

7

37

$\mathrm{P}$

$\mathrm{P}$

le

65

12

17,37

7 


\section{Species}

\section{Conservation \\ status $\dagger$}

Distri-

bution $\div$

Lifeform§

Proposed

reserve

location ๆ

\section{Onagraceae}

Epilobium obscurum

E. pallidiflorum

K

Pittosporaceae

Billardiera alpina

Polygonaceae

Persicaria lapathifolia

$P$. strigosa

Polygonum decipiens

P. plebeium

P. subsessile

Portulacaceae

Calandrinia granulifera

Proteaceae

Banksia integrifolia

Hakea ulicina

Isopogon ceratophyllus

Ranunculaceae

Myosurus minimus

Ranunculus prasinus

$R$. sessiliflorus

Rhamnaceae

Cryptandra amara

Discaria pubescens

Pomaderris elachophylla

$P$. intermedia

P. phylicifolia

Stenanthemum pimeleoides

Spyridium microphyllum

S. obcordatum

S. ulicinum

Rubiaceae

Asperula charophyton

A. scoparia

A. subsimplex

Rutaceae

Phebalium daviesii

Santalaceae

Thesium australe

Sapindaceae

Dodonaea filiformis

Scrophulariaceae

Euphrasia amphisysepala

E. phragmostoma

Euphrasia scabra

E. semipicta

Gratiola pubescens

Veronica distans var. pubescens

$V$. notabilis

V. scutellata

$V$. serpyllifolia

Solanaceae

Solanum opacum

Stackhousiaceae

Stackhousia gunnii

S. pulvinaris

S. viminea
E

K

V

K

K

$\mathrm{X}$

$\mathrm{X}$

E

e

E

V

PV

PV

PV

$\mathrm{P}$

X

$\mathrm{X}$

X

P

V

PV

V

PV

K

V

K

V

X

E le

E

le

le

le
H

H 61

S

3

$\begin{array}{cc}\mathrm{AH} & - \\ \mathrm{AH} & 63 \\ \mathrm{AH} / \mathrm{H} & 34 \\ \mathrm{AH} & - \\ \mathrm{H} & -\end{array}$

ÂH 4

$\mathrm{T}$

$S$

S

11,55

11,42

$\mathrm{AH}$

$\mathrm{H}$

9,20

4,59

AH

1

64

62

11,42

6

$2,6,7$

39

5

6,18

$1,9,31,35$

31

S

$\mathrm{H}$

S

$10,25,32$

H

8,47

8

22

8

$-$

58

33

3

$\mathrm{AH}$

H 1

$\mathrm{H} \quad 3$

H 40 
Species

\section{Conservation \\ status ${ }^{\dagger}$}

Distribution $†$

\section{Proposed \\ reserve} location 9

\section{Sterculiaceae}

Lasiopetalum micranthum Stylidiaceae

Levenhookia dubia

Thymeleaceae

Pimelea axiflora ssp. axiflora

P. filiformis

P. pauciflora

P. phylicoides

P. stricta

Tremandaceae

Tetratheca gunnii

Violaceae

Viola caleyana

Zygophyllaceae

Zygophyllum apiculatum

$\begin{array}{llll}\text { V } & \text { le } & \text { S } & 7,24 \\ \mathrm{X} & & \text { AH } & - \\ \mathrm{V} & & \mathrm{S} & 60 \\ & \mathrm{~S} & 32,71 \\ \mathrm{~K} & \mathrm{~S} & 44 \\ & & \mathrm{~S} & - \\ & & \mathrm{S} & 66\end{array}$

* Including extinct and threatened species.

$\dagger \mathrm{X}=$ extinct, $\mathrm{E}=$ endangered, $\mathrm{V}=$ vulnerable, $\mathrm{K}=$ status unknown, $\mathrm{P}=$ poor $\mathrm{y}$ reserved (for endemic species only).

$\$ \mathrm{e}=$ endemic species, le $=$ local endemic $\left(\right.$ range $<100 \mathrm{~km}^{2}$ ).

$\S \mathrm{AH}=$ annual herb, $\mathrm{AqH}=$ aquatic herb, $\mathrm{H}=$ perennial herb, $\mathrm{CS}=$ climbing shrub, $\mathrm{S}=$ shrub, $\mathrm{T}=$ tree.

I As numbered in figure 2.

TABLE 2

Ratios of Observed to Expected Values for the Native Flora as a Whole

\begin{tabular}{lcccc}
\hline Type of plant & \multicolumn{3}{c}{ Conservation status } & Probability \\
\cline { 2 - 4 } & Unreserved & Threatened* & Extinct & \\
\hline Annuals & 1.47 & 1.77 & 3.61 & P $<0.001$ \\
Herbaceous perennials & 0.64 & 1.27 & 1.37 & n.s. \\
Woody plants & 1.20 & 0.85 & 0.70 & n.s \\
\hline
\end{tabular}

* i.e. endangered and vulnerable.

n.s. = not significant. 
TABLE 3

Areas Most Important for Reservation of Dicotyledonous Angiosperms

Number* Name Species† $\quad$ Land tenure

(1) Tunbridge area

(2)

\begin{abstract}
Barton Road block, Epping Forest
\end{abstract}

(3)

(4)

$$
\begin{aligned}
& \text { Middlesex Plains- } \\
& \text { Vale of Belvoir }
\end{aligned}
$$

Cape PortlandPetal Point

\author{
Mt Vulcan-Dans \\ Hill
}

Prosser River, Orford

Bluemans Creek

Cape Hauy

lagoon, Midlands

Hardings Falls

Cape Barren Island

Meehan Range
Cryptandra amara, Scleranthus diander, Isoetopsis graminifolia, Stackhousia gunnii, Vittadinia gracilis, V. muelleri, $V$. cuneata, Wilsonia rotundifolia, Asperula scoparia. Brachycome rigidula, Calocephalus lacteus, Ptilotus spathulatus, Pultenaea prostrata

Helipterum australe, Pultenaea humilis, Myriophyllum integrifolium, Stenanthemum pimeleoides, Rutidosis multiflora, Glycine latrobeana, Haloragis heterophylla, (Ptilotus spathulatus)

Billardiera alpina, Helipterum albicans, H. anthemoides, Stackhousia pulvinaris, Veronica serpyllifolia

Pultenaea palacea, Brachycome parvula, B. perpusilla, Calandrinia granulifera, Cotula vulgaris,

Gynatrix pulchella, Millotia tenuifolia, Myriophyllum muelleri, Ranunculus sessiliflorus, Hydrocotyle capillaris, Wilsonia humilis, (Rutidosis multiflora), (Myriophyllum integrifolium), (Glycine latrobeana), (Wilsonia rotundifolia)

Epacris virgata, Spyridium obcordatum, Tetratheca gunnit

Scaevola aemula, Helichrysum lycopodiodes, Pomaderris phylicifolia, Acacia axillaris, Spyridium ulicinum, (Stenanthemum pimelioides)

Lasiopetalum micranthum, Helichrysum lycopodioides, Melaleuca pustulata, Eucalyptus barberi, (Stenanthemum pimeleoides)

Euphrasia amphisysepala, E. semipicta, E. phragmostoma

Ranunculus prasinus, (Asperula scoparia), (Calocephalus lacteus)

Pultenaea selaginoides, Callistemon paludosus, Epacris apsleyensis, Dodonaea filiformis, Brachycome sieberi

Bedfordia arborescens, Isopogon ceratophyllus, Hakea ulicina, Leucopogon esquamatus, Pomaderris intermedia, Calystegia marginata, Senecio squarrosus, (Gynatrix pulchella), (Myriophyllum muelleri), (Wilsonia rotundifolia)

Eryngium ovinum, Velleia paradoxa, Eucalyptus risdonii, (Brachycome rigidula), (Millotia tenuifolia), (Vittadinia muelleri), (Ptilotus spathulatus), (Asperula scoparia)
Vacant crown land \& private

Private

Private \& crown land

Private wildlife sanctuary \& coastal reserve

State forest

Private

State forest $\&$ private

Forest reserve

Private

State forest \& forest reserve

Vacant crown land

State recreation area, water catchment, city park, private

* 1-12 in order of priority.

$\dagger()=$ area not chosen for this species 